\title{
Development of TOF-PET detectors based on the Multi-Gap Resistive Plate Chambers
}

\author{
U. Amaldi a , G. Borghi ${ }^{a}$, M. Bucciantonio ${ }^{\text {a }}$, R. Kieffer ${ }^{a}$, J. Samarati ${ }^{a}$, \\ F. Sauli ${ }^{\text {a,* }}$, D. Watts ${ }^{\text {a }}$ \\ a TERA Foundation, Novara, Italy
}

\section{A R T I C L E I N F O}

\section{Article history:}

Received 25 November 2014

Received in revised form

5 January 2015

Accepted 6 January 2015

Available online 13 January 2015

Keywords:

Multi-Gap Resistive Plate Chambers

Positron Emission Tomography

Time of Flight

\begin{abstract}
A B S T R A C T
We describe the development, construction and preliminary results obtained with medium-size MultiGap Resistive Plate Chambers prototypes designed to detect and localize $511 \mathrm{keV}$ photons for Positron Emission Tomography imaging applications. The devices are intended for in-beam monitoring of the treatment plans throughout deep tumor therapy with hadron beams; emphasis is put on achieving coincidence time resolutions of few hundred ps, in order to exploit optimized reconstruction algorithm and reduce the heavy non-correlated background contributions distinctive of this operation. Using technologies developed for high energy physics experiments, the detectors can be built for covering large areas, thus leading the way to the conception of full-body PET systems at low cost.
\end{abstract}

(c) 2015 Elsevier B.V. All rights reserved.

\section{Introduction}

Positron Emission Tomography (PET) performed with the injection of site-specific positron-emitter tracers in the patient is one of the most powerful diagnostic techniques employed to localize invasive neoplasms. With the on-going commissioning of dedicated tumor treatment centers exploiting ion beams for patient irradiation (hadrotherapy), a need has aroused to develop in-beam imaging and diagnostic techniques which, adding to conventional X-ray radiography, Positron Emission Tomography and Computer Assisted Tomography, aim at improving the patient quality assurance during or shortly after the treatments.

One of the modalities under scrutiny is the use of dedicated PET systems capable of detecting the two collinear gammas emitted by the decay of isotopes with positron activity, created by the beamtarget interactions during the exposure. With suitable data reconstruction and calibration algorithms, the 3-D mapping of the local positron activity provides information both on the position and the dose of the irradiation. The additional recording of the time difference between the two gammas in each event, the so-called Time-of-Flight (TOF)-PET, permits to restrict the region of interest, thus improving the quality of the signal in presence of the heavy non-correlated background created during the irradiation; it is also important to reduce the relevance of the artifacts generated by the incomplete

\footnotetext{
* Corresponding author at: CERN, Geneva, Switzerland.

E-mail address: fabio.sauli@cern.ch (F. Sauli).
}

angular coverage of the detectors, having openings to prevent being hit by the beam.

In the framework of the European FP7 Collaborative Project ENVISION (European NoVel Imaging Systems for ION therapy), several TOF-PET approaches have been studied, based on the use of scintillators with very fast readout, or on the use of Multi-Gap Resistive Chamber detectors; the latter development is described in this note. A comparative study of the two approaches can be found in Ref. [1].

\section{Multi-Gap Resistive Plate Chambers}

Resistive Plate Chambers (RPCs) are widely used tools for detection and localization of fast charged particles in High Energy Physics experiments, covering large areas of sensitivity at low cost. An improved version of the device, the Multi-Gap RPC (MRPC) has been developed specifically to provide a more precise TOF measurement, and is exploited for particle identification $[2,3]$.

A four-gap MRPC, schematically shown in Fig. 1, consists of a stack of thin glass electrodes encompassing several gas-filled gaps; internal insulating support lines ensure the mechanical gap uniformity. A moderate resistivity layer added on the outer sides of the stack permits to apply a difference of potential between the outer electrodes, creating a high electric field through the structure.

The high field value provokes the formation of electron-ion avalanches between the electrodes, initiated by the release of ionization by charged particles crossing the structure; the movement of electrons created in the exponentially growing avalanche induces 


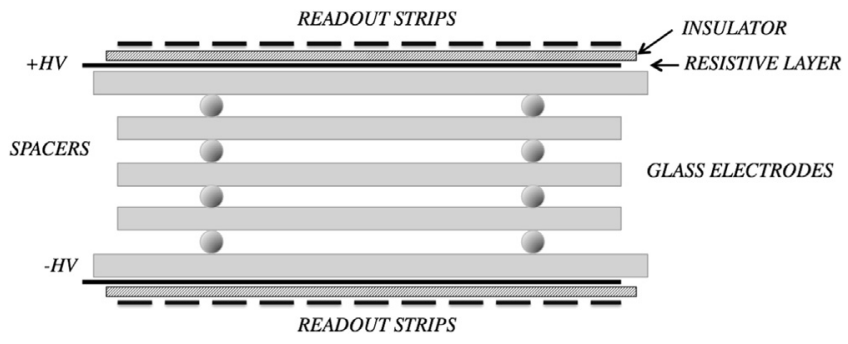

Fig. 1. Schematics of a four-gap MRPC.

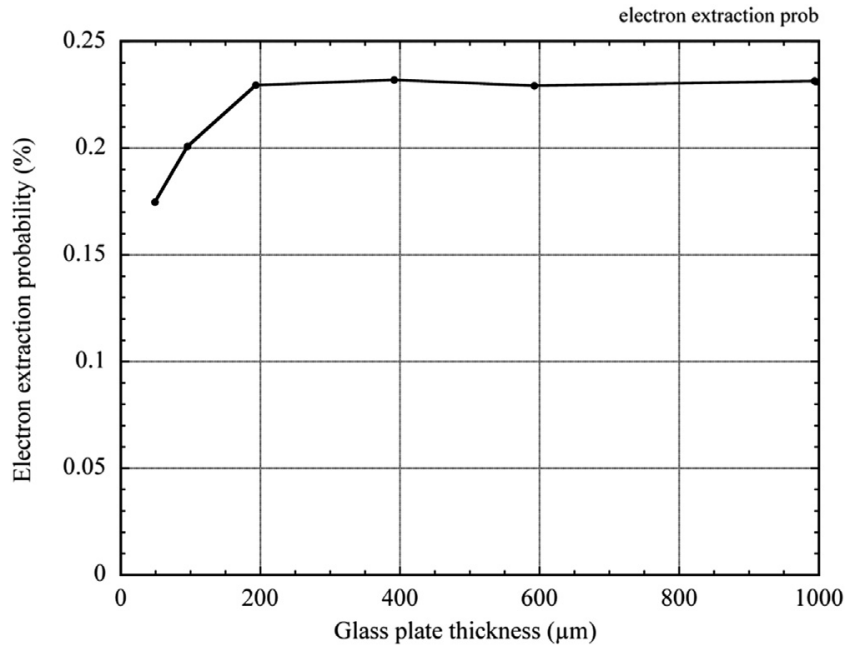

Fig. 2. Probability for a secondary electron to be created in the glass plate by $511 \mathrm{keV}$ photons and emerge in the gas gap as a function of the plate thickness.

through the resistive plates fast signals on external pick-up electrodes insulated from the resistive coatings. The best timing is obtained using very thin gas gaps, minimizing the fluctuations in the avalanche development; the multiple structures with several thin gas gaps in a stack compensate for the reduced efficiency of a single layer. The signals induced on the two sides of the detector are equal and opposite in polarity; use of a front-end amplifier with differential inputs connected to facing strips ensures the best signal over noise ratio and timing performances. Use of soda-lime glass with moderate conductivity causes a redistribution of the internal charges after application of the external voltage and results in the application of high and uniform electric fields across the gas gaps. The assembly is encapsulated in a gas containment box, provided with the feedtrough needed to apply the high voltage and extract the signals.

To ensure a proper voltage distribution and a transparency to the signals through the stack, the resistivity of the glass is chosen in the range $10^{9}-10^{12} \Omega \mathrm{cm}$; typical values for the surface resistivity of the external resistive layers used to distribute the voltage are around $10^{5}-10^{6} \Omega$ /square. Since the best performances are obtained at the highest values of electric fields, non-flammable gas fillings with high dielectric rigidity are preferred, such as tetrafluoroethane $\left(\mathrm{C}_{2} \mathrm{~F}_{4} \mathrm{H}_{2}\right)$, pure or with the addition of small percentages of isobutane, carbon dioxide or carbon tetrafluoride [4-6]; its high density $\left(4.2 \times 10^{-3}\right)$ results in a large ionization yield, enhancing the detection efficiency for charged particles.

Widely used in High Energy Physics (HEP) experiments, MRPCs provide full detection efficiency and time resolutions better than 50 ps rms for relativistic charged particles $[7,8]$.

\section{Detection of $511 \mathrm{keV}$ photons}

Several works have described the application of the technology in the detection of hard photons, and in particular the $511 \mathrm{keV}$ gammas used for PET [9-14]. While for the detection of charged particles the glass plates serve only the role of field-providing electrodes, in the detection of neutral radiation they act also as converters. A secondary electron, created by an interaction within the plate, scatters losing energy through the material until eventually emerging in the gas layer, where it produces a detectable ionization trail; its energy is generally insufficient to cross over to a second gap. For charged particles, avalanches develop in several gaps and the induced signals add-up improving efficiency and time resolution. For photons, instead, only one gap is interested by the counting process; charge signals are consequently smaller, adversely affecting the time resolution and signal/noise ratio for photons as compared to the detection of charged tracks. This has motivated a thorough simulation work using the GEANT4 toolkits [15] to help the optimization of the detector design and operating conditions.

To study the effect on detection efficiency of the glass and gas gap thickness, we have simulated the interaction process on a single cell, consisting of a glass plate and two gas gaps on each side of the converter. In glass, the interaction is almost entirely due to Compton processes. The photon absorption probability increases with the converter thickness; however, the short range in the material of the released secondary electrons has as consequence that only a layer of converter comparable with the electron range is effective, which results in reaching an almost constant detection probability above a certain plate thickness. For $511 \mathrm{keV}$ gammas perpendicular to the soda-lime glass plate (density $2.4 \mathrm{~g} \mathrm{~cm}^{-3}$ ), Fig. 2 shows that the limit is reached at around $200 \mu \mathrm{m}$.

Multiple Coulomb interactions scatter the secondary electron in the converter's medium, resulting in a gradual loss of energy and a deviation from the original trajectory. The computed distribution of residual energy and angular distribution around the incident photon direction of the electrons emerging into the gas gap are shown in Figs. 3 and 4 for a $400 \mu \mathrm{m}$ glass plate. The rather narrow width of the angular distribution helps reducing the error in the localization of the photon through the detection of the signal produced by the ionization in the gas. The simulation results indicate also that a non-negligible fraction of the secondary electrons, around $10 \%$, emerges backwards into the upper gas layer, adding to the detection efficiency in a multigap device. Note also that electrons in lower energy part of the distribution have larger ionization losses, easing detection.

The small value of the single-layer efficiency, $0.23 \%$, requires the use many layers to reach an acceptable detection efficiency. For a multigap structure, the gradual removal of photons from the

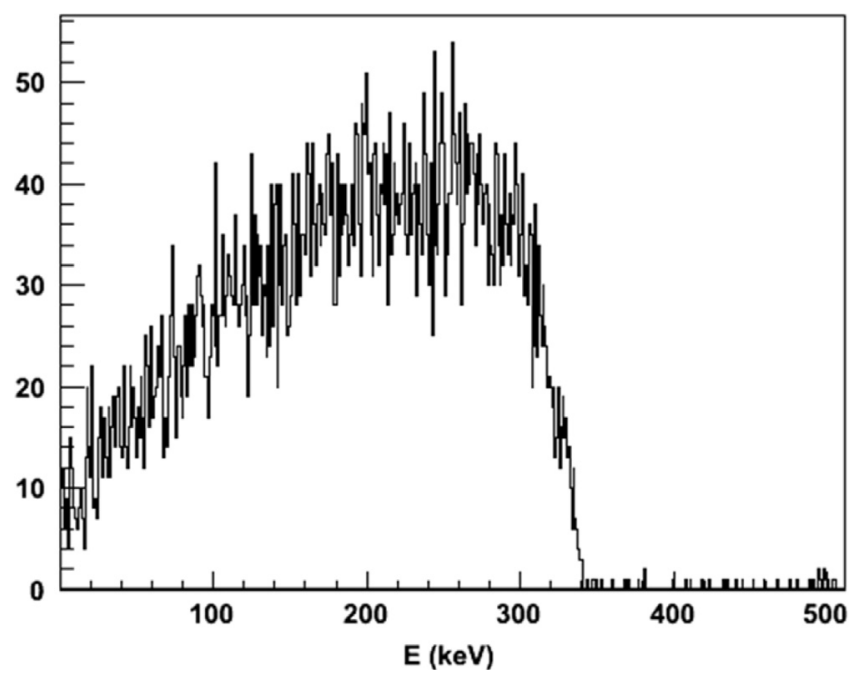

Fig. 3. Residual energy distribution of secondary electron resulting from the interaction of a $511 \mathrm{keV}$ photon in the glass and emerging in the gas gap in the forward direction. 


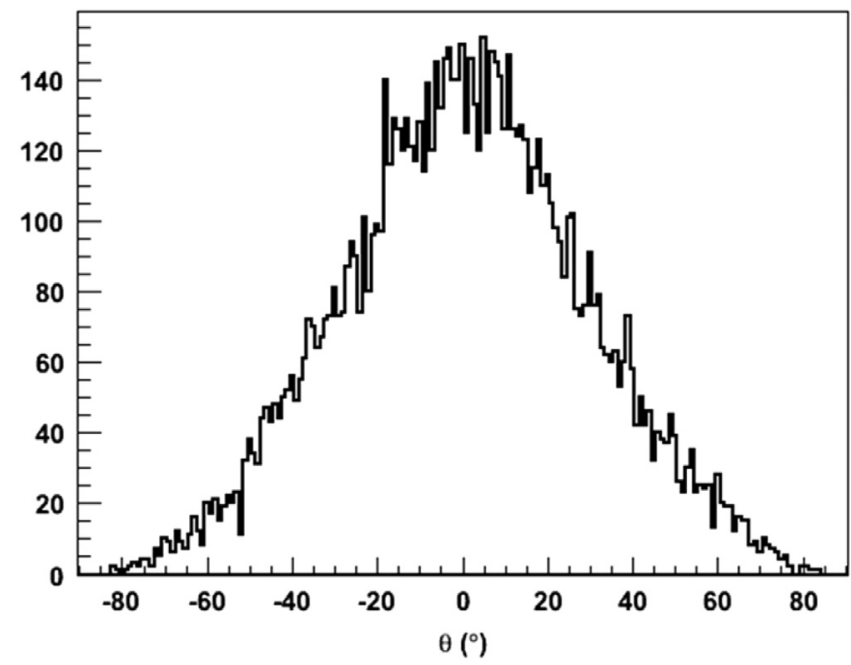

Fig. 4. Angular distribution of the forward secondary electrons emerging in the gas gap.

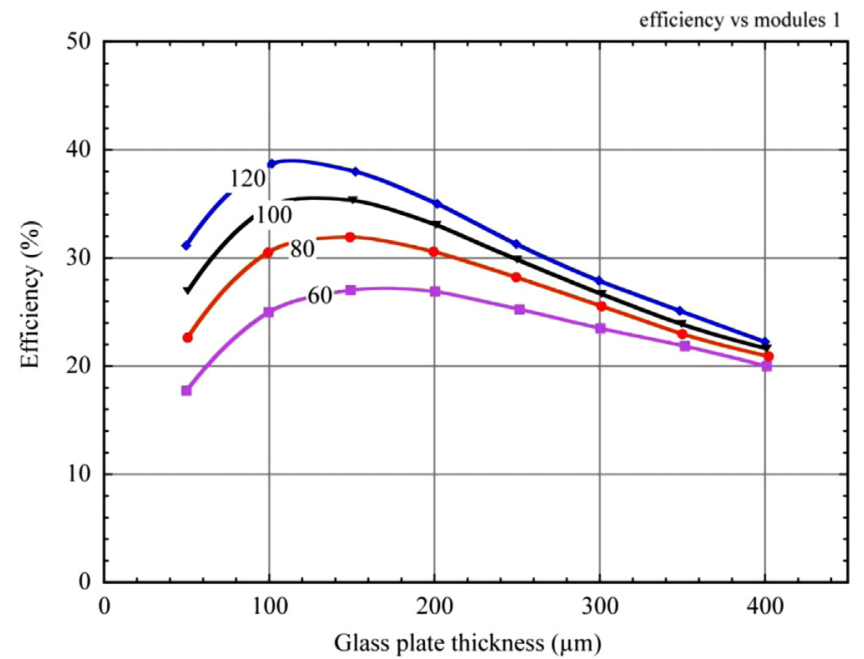

Fig. 5. Computed detector efficiency as a function of glass thickness and number of 4-gap modules.

beam results in a decrease of the extraction efficiency above an optimum value of thickness that depends on the number of layers, as shown in Fig. 5, computed for increasing numbers of identical four-gap modules. The use of lead glass plates as electrodes, providing higher conversion efficiency, has been considered, but is hindered by the scarce availability of the material in thin layers and by its excessively high resistivity.

\section{Choice of materials and geometry}

Following the original developments of the MRPC [4], we have used for all our detectors a $400 \mu \mathrm{m}$ thick, floated soda-lime glass of various sizes, having a nominal resistivity around $10^{12} \Omega \mathrm{cm}$. ${ }^{1}$ The low atomic number of the constituents requires, to reach detection efficiencies useful for PET applications, to use several plates in a module, and stacks of many modules. While improving the conversion efficiency, the increase of the number of glass plates with moderate conductivity within a module influences the process of charge induction on the external pick-up electrodes, affecting both

\footnotetext{
${ }^{1}$ VERTEC 400, AGC Glass Europe.
}

the amplitude and the extension of the signals. The process has been studied experimentally with a mock-up setup consisting in a stack with a variable number of glass plates. A fast signal could be injected with a $2 \mathrm{~mm}$ wide strip laid over the first glass in the stack, and the induced charge profile recorded in a set of parallel pick-up strips, $400 \mu \mathrm{m}$ apart, externally in contact with the last glass plate; the point at zero has been measured with only a thin insulator between the pulsing strip and the readout. Fig. 6 shows the measured total signal amplitude and the signal width, or cluster size, as a function of the number of glass plates between the injection and pick-up electrodes; as expected, with the increase in the number of plates, the induced signal gets smaller and wider. A four-gap module (five glass plates) appears to be a reasonable choice for optimum performance.

The uniformity and resistivity of the layers used to distribute the voltage affect the amplitude of the signal induced on the external pick-up electrodes. A variety of methods have been described to produce thin layers of controlled resistivity $[16,17]$. The most common deposition method makes use of a colloidal graphite emulsion, applied to the glass and dried under controlled conditions; we have tested values of surface resistivity up to $1 \mathrm{M} \Omega$ /square, which can be manufactured with reasonable uniformity. ${ }^{2}$ A more recent development makes use of $25 \mu \mathrm{m}$ thick foils of high resistivity polymers, typically of a few $\mathrm{M} \Omega /$ square, pasted or laminated to the glass. ${ }^{3}$

Measured with a fully operational, four gap MRPC, Fig. 7 shows the cluster size of the induced signals in a wide range of values of the external resistive layers; the point at the highest value corresponds to the surface resistivity of bare glass, and has been measured with the (grounded) readout strips in contact with the glass [18]. As expected, high resistivity provides narrower signals; values above $1 \mathrm{M} \Omega$ /square are however rather unstable and difficult to manufacture. For lowrate PET applications, a larger cluster size is in fact an advantage, as it permits to use wider readout strips, decreasing costs, still with good localization properties exploiting a center-of gravity algorithm.

\section{MRPC design for PET}

The standard design of MRPC detectors developed for particle physics experiments, employing sturdy frames and gas containment boxes, is not suitable for PET applications due to absorption losses in the inert materials and to an unfavorable aspect ratio (active to total sensitive area). We have developed a more suitable MRPC assembly, shown schematically in Fig. 8 using a thin fiberglass supporting frame, glued to the first outer glass electrode $[18,19]$. The internal electrodes, smaller in size, pile up within the frame, separated by insulating spacers; the assembly is completed pasting the last electrode on the top. Miniature tubes on opposite sides of the frame provide the gas input and output. A matrix of insulating cylindrical pillars, $300 \mu \mathrm{m}$ in diameter and $300 \mu \mathrm{m}$ high, at $10 \mathrm{~mm}$ spacing, built on the glass plates prior to installation with photolithographic processing guarantees the gap uniformity while permitting the circulation of the filling gas (Figs. 9 and 10). ${ }^{4}$ The detector assembly is carried out in clean room conditions, after thorough cleaning of all components.

A medium size prototype $\left(90 \times 65 \mathrm{~mm}^{2}\right)$ built using the described technology is shown in Fig. 11, with the readout stripped electrodes covering the all active area; Fig. 12 shows instead a complete MRPC detector, with the front-end electronics mounted on both sides of the readout strips, the high voltage connections and gas inlets. Used for most of the measurements, the module has eight signal pick-up strips on each side, $3.5 \mathrm{~mm}$ wide at $4 \mathrm{~mm}$ pitch; as discussed in the next section, the amplifier-discriminator

\footnotetext{
${ }^{2}$ Kindly provided by G. Mikenberg, The Weizmann Institute of Science.

${ }^{3}$ Dupont Kapton Type XC.

${ }^{4}$ Technology developed by R. De Oliveira, CERN PH-DT-DD.
} 


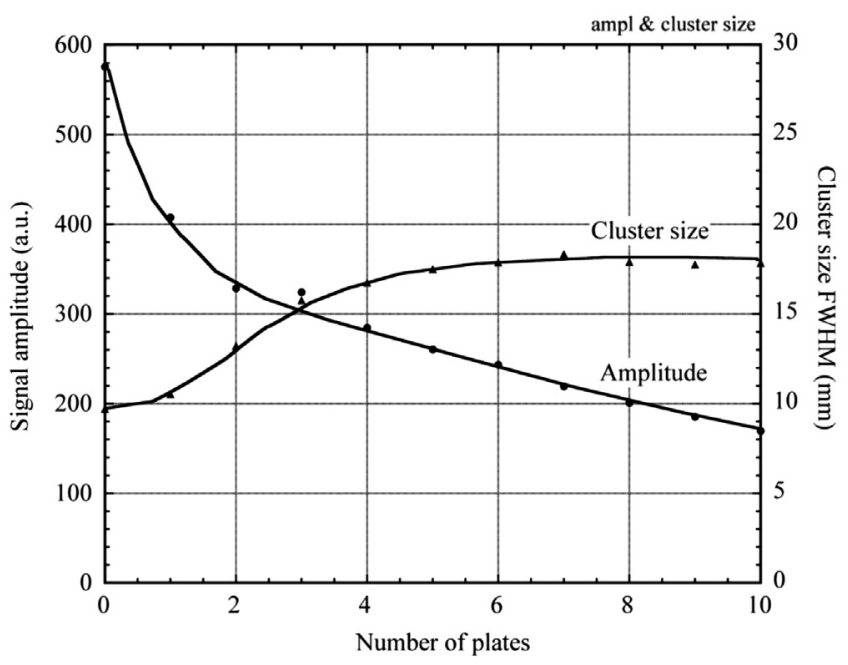

Fig. 6. Induced signal amplitude as a function of the number of glass plates.

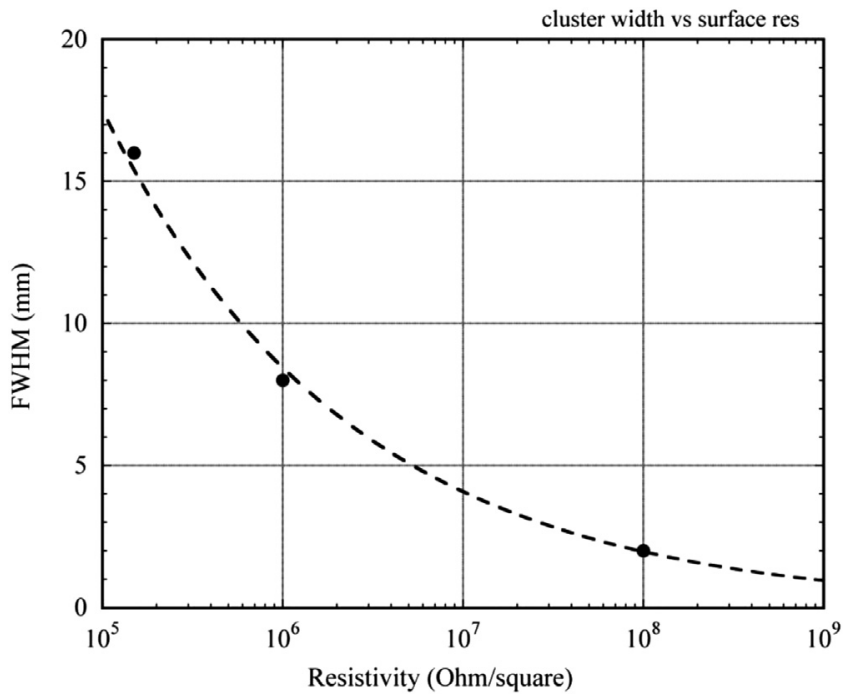

Fig. 7. Induced signal cluster size for several values of the external resistive layer.

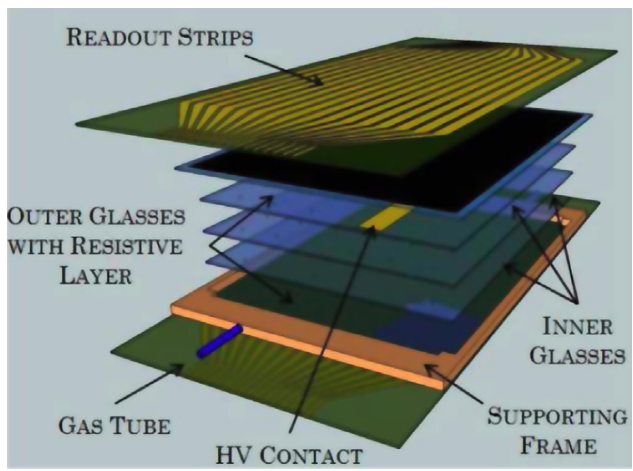

Fig. 8. Schematics of the TERA four-gap MRPC

is used in a differential mode, with the two inputs connected to a facing pair of readout strips on the two sides of the detector.

The manufacturing method described above permits the realization of large area detectors at low cost, limited only by the availability of components. Fig. 13 shows a larger module, with $300 \times 120 \mathrm{~mm}^{2}$ active area, during construction; while there were no particular manufacturing problems, a poor manipulation resulted in a shattered glass and the module could not be tested.

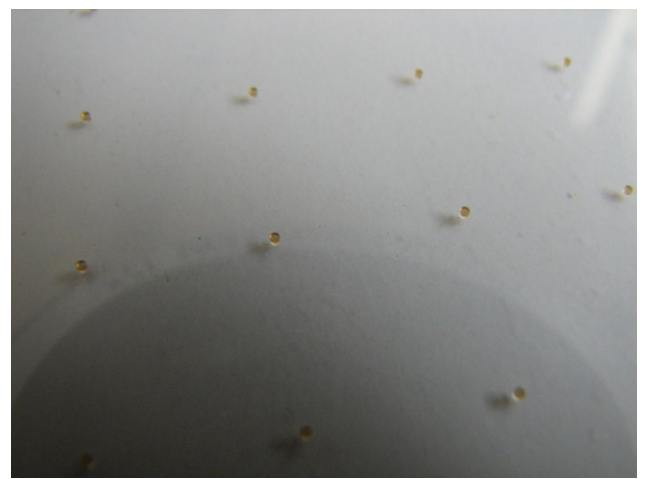

Fig. 9. The matrix of insulating pillars on glass, $300 \mu \mathrm{m}$ high and $10 \mathrm{~mm}$ apart, ensuring the gap uniformity.

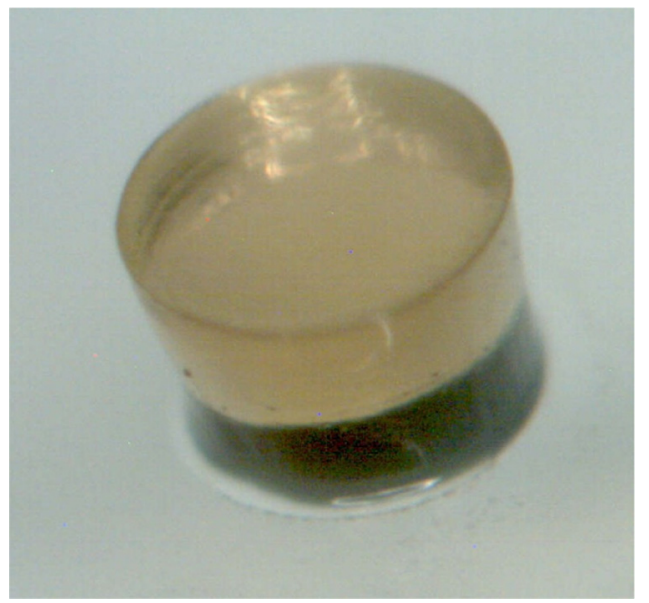

Fig. 10. Close view of an insulating pillar, $300 \mu \mathrm{m}$ in diameter.

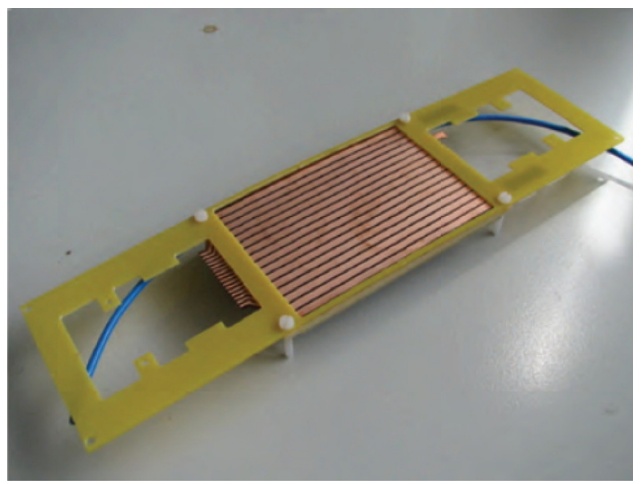

Fig. 11. An assembled MRPC, with $90 \times 65 \mathrm{~mm}^{2}$ active area.

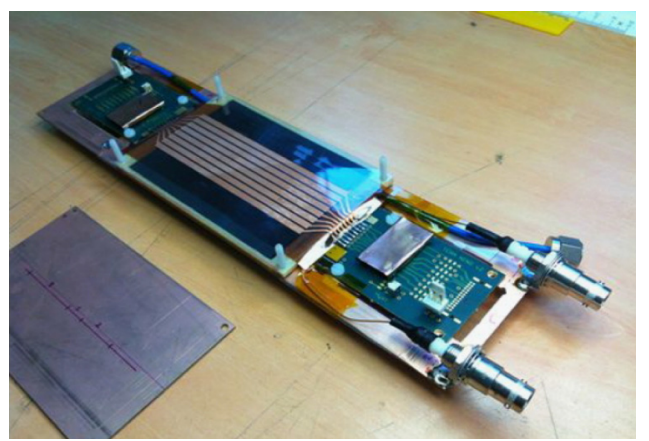

Fig. 12. The complete MRPC prototype with the front-end electronics, gas and HV inlets; signals are read-out from eight strips on each side, connected to the differential inputs of the amplifiers. 


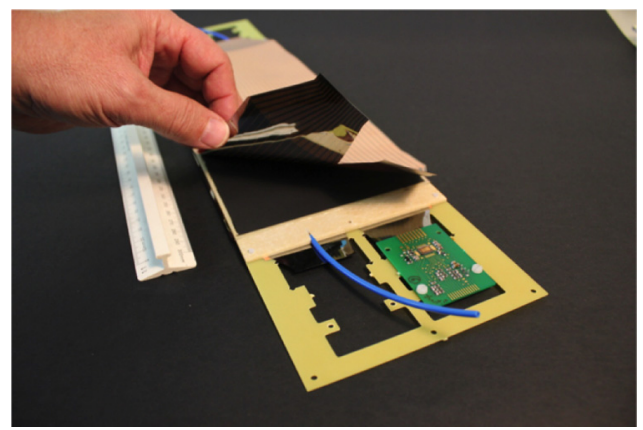

Fig. 13. A large MRPC prototype during construction.

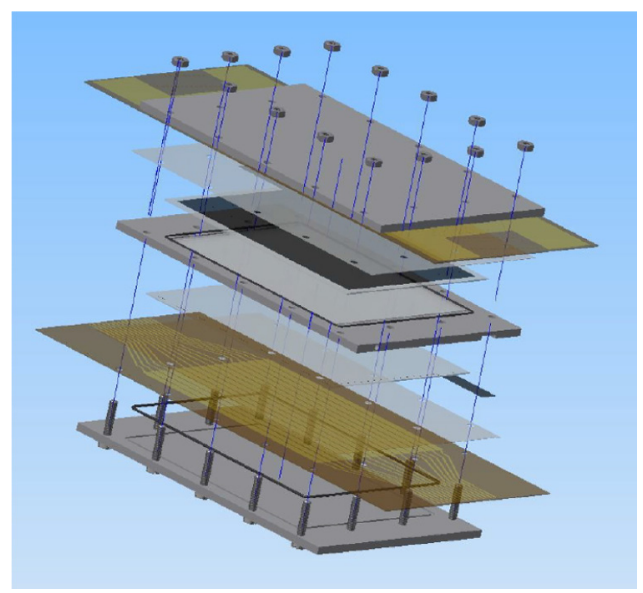

Fig. 14. A dismountable MRPC assembly.

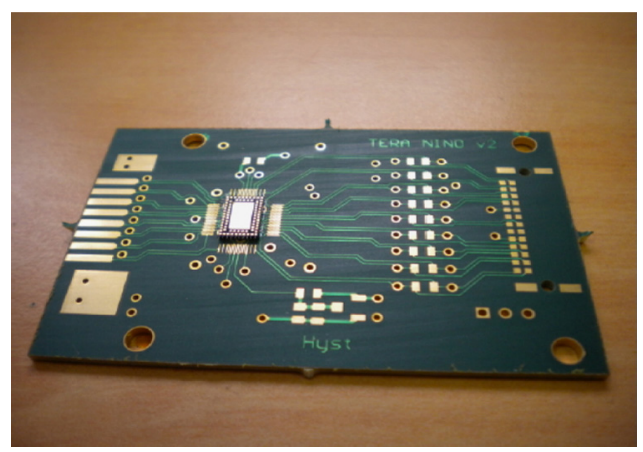

Fig. 15. 8-channel front-end card.

In the course of the research on MRPC detectors, and in order to have flexibility in the replacement of the parts and to correct local imperfection, we have also developed the dismountable assembly shown in Fig. 14; the components are identical to those described above. The gas tightness is provided by miniature rubber O-rings between frames and an array of insulating nuts and bolts, visible in the figure. Both the compact and the dismountable assembly modules have been used for the measurement, without noticeable difference in the performances.

\section{Front-end electronics}

A custom readout board (Fig. 15) has been designed and implemented at both ends of the strips, based on the NINO ASIC, an 8-channel fast differential amplifier-discriminator developed at CERN for high energy physics [20]. Signals from pairs of corresponding

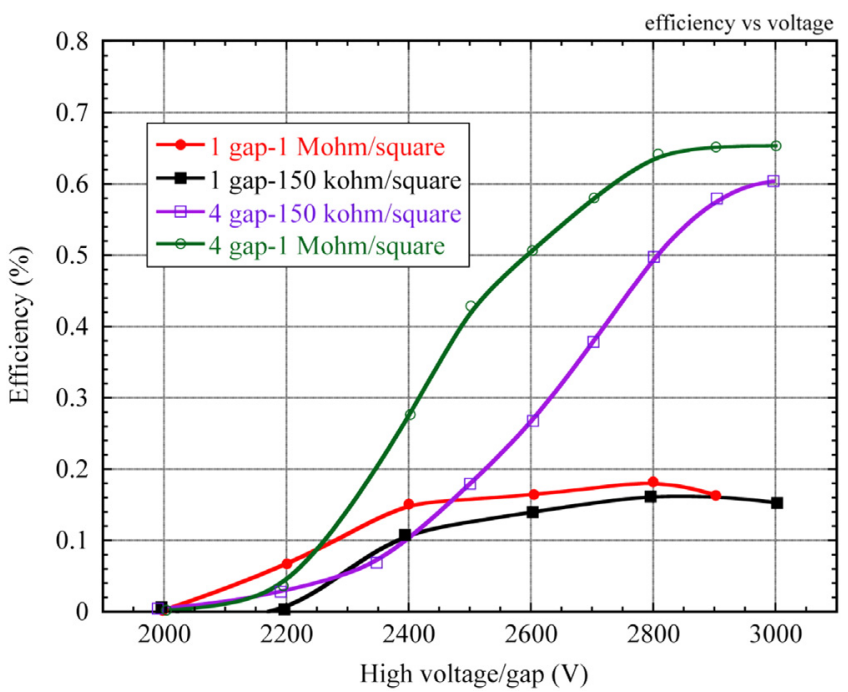

Fig. 16. Measured efficiency for $511 \mathrm{keV}$ photons for single-and four-gap MRPC and two values of the resistive coatings.

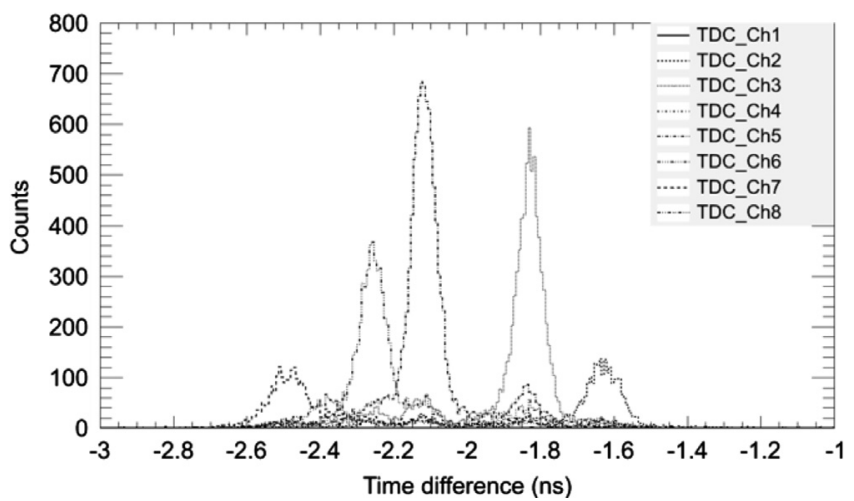

Fig. 17. Distributions of the time difference between the two ends of eight adjacent strips, for a collimated $511 \mathrm{keV}$ source.

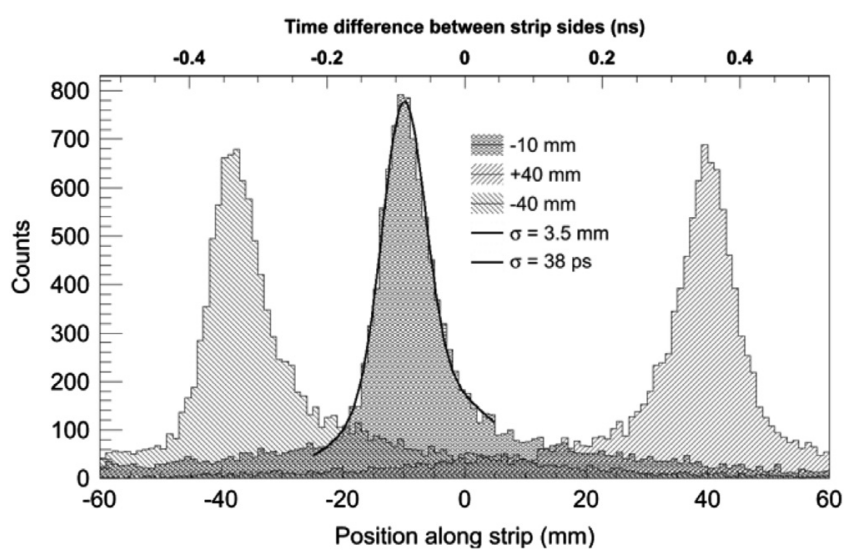

Fig. 18. Distributions of the measured longitudinal time and space dispersions for three positions of the collimated source along the strips.

strips on each side of the detectors are brought to the differential inputs of the amplifiers mounted on the cards. The outputs produce discriminated LVDS pulses, with a rising edge close to $1 \mathrm{~ns}$ and a width corresponding to the time over threshold of the input signals.

The information is used to correct for the dispersive effect of the amplitude-dependent triggering time, or slewing, essential to achieve the best timing performances [4]. All channels are individually 


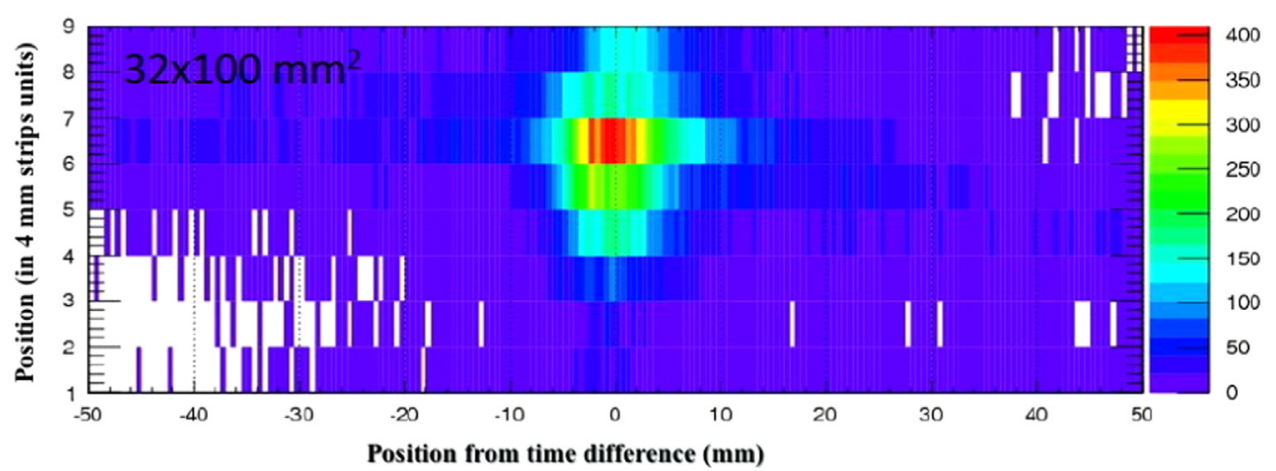

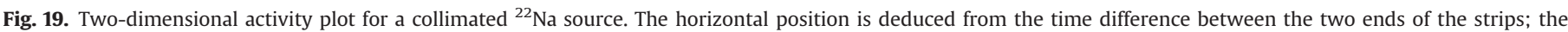
vertical is the strip number (in $4 \mathrm{~mm}$ units).

connected to the latched entries of an FPGA to determine which channel is fired; a fast-OR output provides a logical signal if any of the eight inputs is above threshold. As the fast electronics system foreseen to record the TOF on individual channels was not yet available for the present work, a time difference measurement between detectors was performed on the fast-OR outputs using a 4-channel, $3.5 \mathrm{GHz}$ bandwidth oscilloscope; data are merged into a dedicated Labviewbased acquisition system, saved on disk and subsequently analyzed.

\section{Detection efficiency for $511 \mathrm{keV}$ photons}

The MRPC photon detection efficiency has been measured as a function of operating voltage inserting a ${ }^{22} \mathrm{Na}$ positron-emitting source between the detector and a reference counter, a $10 \mathrm{~mm}$ diameter, 1 in. thick BGO scintillator with conventional photomultiplier readout; detector and scintillator were placed about $10 \mathrm{~cm}$ from the source. A selection of events in the $511 \mathrm{keV}$ photo-peak recorded with the scintillator and an additional geometrical collimation with lead absorbers ensured that most of the events are genuine collinear $\gamma \gamma$ decays. The efficiency, defined as the ratio of coincidences between scintillator and detector and photopeak counts in the scintillator, has been measured with two MRPC configurations, namely single- and four-gap, and two values of resistivity of the external resistive coating used to apply the voltage (150 $\mathrm{k} \Omega$ /square and $1 \mathrm{M} \Omega /$ square). The results are summarized in Fig. 16, as a function of the voltage on each gap. ${ }^{5}$ For a single gap, the measured value corresponds well to the computed result, Fig. 2; the four-gap value, four times larger, confirms that there are no signal losses due to the interaction depth.

\section{Timing calibrations and determination of the longitudinal coordinate}

Due to variations in the input capacitance and connection length, and in the response of the NINO circuit, the time difference between the two ends of a readout strip for a $511 \mathrm{keV}$ source, collimated in a direction perpendicular to the strips, differs by a non-negligible fraction of a ns as shown in Fig. 17 for eight adjacent strips. Fluctuations in the discriminators and measurement setup and reflections at the two ends of the lines contribute to the dispersions. As the same signal is detected on the two sides of each strip, the effect of the discriminators time walk is

${ }^{5}$ As discussed above, it is assumed that shortly after application of the voltage, the redistribution of charges in the glass plates makes them equipotential; the voltage applied on each gap is then the total HV divided by the number of gas gaps. minimized, thus providing a result in first approximation independent from the pulse amplitude.

After calibration, the differences can be compensated, resulting in an overall distribution with a width of $38 \mathrm{ps}$ rms, shown by the central distribution in Fig. 18 that includes the width of the source $(\sim 2 \mathrm{~mm})$.

Displacing the collimated source along the strips' direction, one can deduce the longitudinal position from the time difference between the two ends. As shown in Fig. 18 for three positions at $-40,-10$ and $10 \mathrm{~mm}$ from the center, the propagation velocity along the strips is $225 \mathrm{~mm} / \mathrm{ns}$; the width of the distributions determines a longitudinal position accuracy of $3.5 \mathrm{~mm}$ rms.

A two-dimensional image of the source, obtained exposing the center of the detector through a $2 \mathrm{~mm}$ slit collimator is shown in Fig. 19; the longitudinal coordinate is deduced from the time difference between the two ends of the strips, while the transverse coordinate is given by the position of the triggered strip.

\section{Coincidence Time Resolution}

The Coincidence Time Resolution (CTR) has been measured recording on the fast oscilloscope the signals from both sides of two identical detectors on each side of the source (Fig. 20); to reduce geometrical dispersions, a narrow slit lead collimator limited the exposure to a central region of the chambers, and a single strip was connected to the recorder. This choice resulted in a very low coincidence rate (below $\mathrm{Hz}$ ), which induces the appearance of instabilities in the detector operation, and has affected the results. The recorded data were analyzed averaging the information from the two ends of each detector to correct for the position dependence of the time; however, due to a problem with the signals shape, probably due to reflections and impedance mismatching, the time slewing correction could not be performed affecting the results. Fig. 21 shows an example of the time difference between the two detectors, and has a width of $240 \mathrm{ps} r \mathrm{rs}$; under the assumption of equal dispersions in the two detectors, this corresponds to a single detector resolution of $170 \mathrm{ps}$ rms.

\section{Conclusions and summary}

Multigap Resistive Plate Chambers of various designs and sizes have been built and tested, in view of their possible use as detectors for Positron Emitter Tomography; emphasis has been put on finding manufacturing methods appropriate for this application, requiring the optimization of the ratio between active and total area, and minimizing the absorption losses in framing materials. With a fourgap module we have demonstrated a detection efficiency for $511 \mathrm{keV}$ photons around $0.7 \%$, consistent with a Monte Carlo simulation. 


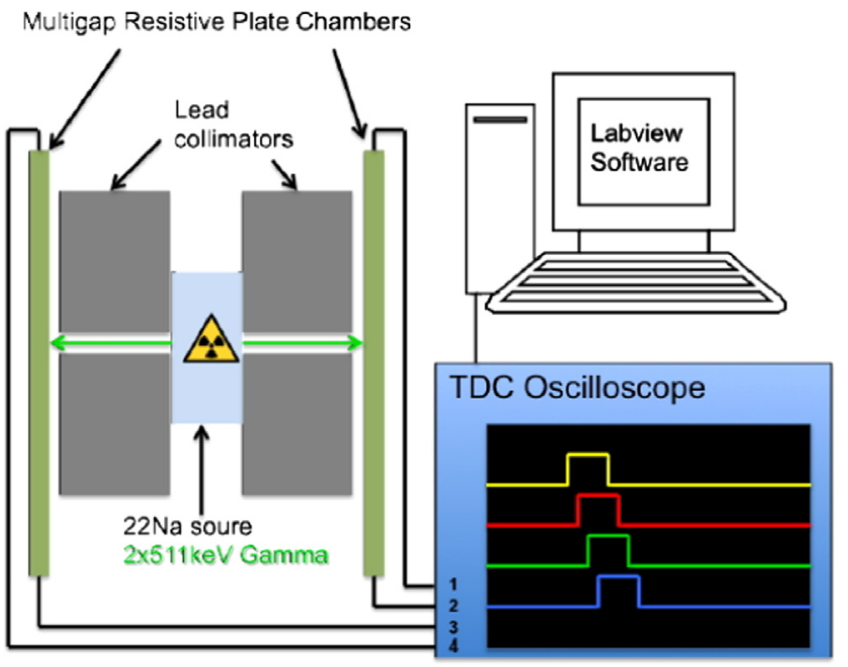

Fig. 20. Setup for the measurement of the TOF resolution.

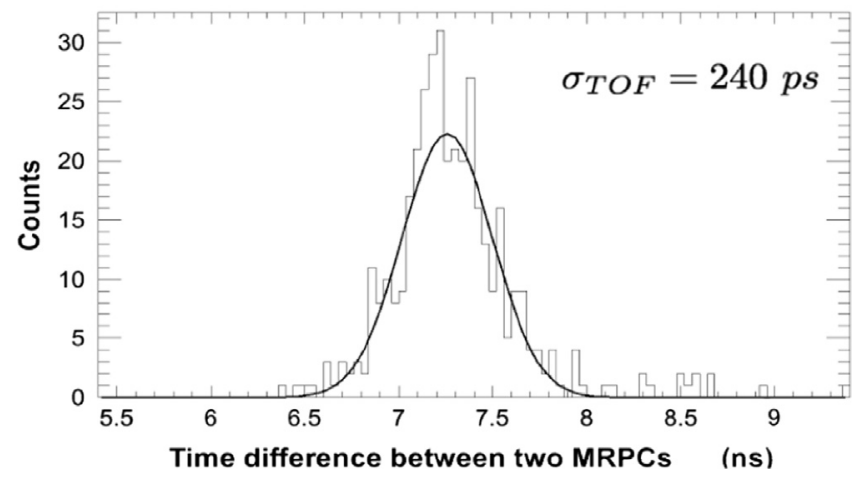

Fig. 21. Coincidence TOF resolution. The corresponding single MRPC resolution is 170 ps rms.

Signals are detected on both sides of the detectors on sets of parallel pick-up strips, connected to differential fast amplifier-discriminators. Recording the time difference between the two ends of the strips permits to determine the longitudinal position of a collimated ${ }^{22} \mathrm{Na}$ source with an accuracy of $4 \mathrm{~mm}$ rms, the transverse coordinate being defined by the strips' pitch $(4 \mathrm{~mm})$. Using a preliminary electronics setup, not permitting the amplitude-dependent time slewing correction, we have achieved a Coincidence Time of Flight resolution of $240 \mathrm{ps}$ rms.

\section{Acknowledgments}

The help of Crispin Williams (CERN-PH-AIO) along the development work, as well as for the procurement of the specialty glass and the front-end electronics is acknowledged.
The technology for the lamination of the conductive polymer resistive layers and of the insulating pillars spacers has been developed by Rui de Oliveira at CERN's detector technologies group.

The project has been partly supported by the European ENVISION Program Grant Agreement no 241851 and by the Marie Curie Early Initial Training Network Fellowship of the European Community Seventh Framework Programme PITN-GA-2008-215840PARTNER and FP7 Grant Agreement no. 264552 ENTERVISION. D. Watt was PARTNER fellow; R. Kieffer was ENTERVISION fellow.

\section{References}

[1] F. Diblen, H. Rohling, I. Torres-Espallardo, P. Solevi, J. Gillam, D. Watts, S. Espana, S. Vandenberghe, F. Fielder, M. Rafecas, IEEE Nuclear Science Symposium Conference Records, 2012, p. 2212.

[2] E. Cerron-Zeballos, I. Crotty, D. Hatzifotiadou, J.L. Valverde, M.C.S. Williams, A. Zichichi, Nuclear Instruments and Methods A 374 (1996) 132.

[3] P. Fonte, A. Smirnitski, M.S.C. Williams, Nuclear Instruments and Methods A 443 (2000) 201.

[4] A. Akindinov, F. Anselmo, M. Basile, A. Cerron Zeballos, L. Cifarelli, et al., Nuclear Instruments and Methods A 456 (2000) 16.

[5] A.N. Akindinov, A. Alici, P. Antonioli, S. Arcelli, M. Basile, G.C. Romeo, L. Cifarelli, et al., Nuclear Instruments and Methods A 532 (2004) 562.

[6] L. Lopes, P. Fonte, A. Mangiarotti, Nuclear Instruments and Methods A 661 (2012) S194.

[7] A. Akindinov, A. Alici, P. Antonioli, S. Arcelli, Y.W. Baek, et al., Nuclear Instruments and Methods A 602 (2009) 709.

[8] A. Akindinov, A. Alici, P. Antonioli, S. Arcelli, M. Basile, F. Bellini, et al., Nuclear Instruments and Methods A 661 (2012) S98.

[9] A. Blanco, V. Chepel, R. Ferreira-Marques, P. Fonte, M.I. Lopes, V. Peskov, A. Policarpo, Nuclear Instruments and Methods A 508 (2003) 88.

[10] A. Blanco, M. Couceiro, P. Crespo, N.C. Ferreira, R. Ferreira Marques, P. Fonte, L. Lopes, J.A. Neves, Nuclear Instruments and Methods A 602 (2009) 780.

[11] N. Ilieva, V. Kozuharov, I. Lessigiarska, L. Litov, B. Pavlov, P. Petkov, AIP Conference Proceedings, 1203, 2010, p. 820.

[12] K. Doroud, D. Hatzifotiadu, S. Li, M.S.C. Williams, A. Zichichi, R. Zuyeuski, Nuclear Instruments and Methods A 660 (2011) 73.

[13] A. Tamborini, Nuovo Cimento C 34 (2011) 185.

[14] A. Banerjee, A. Roy, S. Biswas, S. Chattopadhyay, G. Das, S. Pal, Nuclear Instruments and Methods A 718 (2013) 138.

[15] S. Agostnelli, J. Allison, K. Amako, J. Apostolakis, et al., Nuclear Instruments and Methods A 506 (2003) 250.

[16] C. Beard, R. Hammarström, D. Hatziofotiadou, C. Kleinwort, L. Köpke, et al., Nuclear Instruments and Methods A 286 (1990) 117.

[17] M. Bedjidian, J.-P. Ianigro, R. Kieffer, I. Laktineh, N. Lumb, S. Vanzetto, Nuclear Instruments and Methods A 623 (2010) 120.

[18] G. Borghi, Development of a Time-Of-Flight Positron Emission Tomography System for Dose Monitoring in Hadrontherapy (Thesis), Università MilanoBicocca, Milano, Italy, 2011.

[19] D. Watts, G. Borghi, F. Sauli, U. Amaldi, Journal of Radiation Research 54 (2013) 1136.

[20] F. Anghinolfi, P. Jarron, A.N. Martemiyanov, E. Usenko, H. Wenninger, M.S. C. Williams, A. Zichichi, Nuclear Instruments and Methods A 533 (2004) 183. 